\title{
Host-associated differences in morphometric traits of parasitic larvae Hirsutiella zachvatkini (Actinotrichida: Trombiculidae)
}

\author{
Hanna Moniuszko ${ }^{1} \cdot$ Grzegorz Zaleśny $^{1} \cdot$ Joanna Mąkol $^{1}$
}

Received: 10 February 2015/Accepted: 30 April 2015/Published online: 23 May 2015

(C) The Author(s) 2015. This article is published with open access at Springerlink.com

\begin{abstract}
Examination of host-associated variation in the chigger mite Hirsutiella zachvatkini (Schluger) revealed morphological differences among larvae infesting sympatric hosts: Apodemus agrarius, Apodemus flavicollis and Myodes glareolus. The analysis included 61 variables of larvae obtained from their gnathosoma, idiosoma and legs (measurements and counts). Statistically significant differences were observed for metric characters of the legs as opposed to the scutum. In view of the conspecificity of the mites, supported by comparison of COI gene products obtained from larvae and laboratory-reared deutonymphs, the observed variation is attributed to phenotypic plasticity. The knowledge of larval morphology, including intraspecific variation of metric characters, supported by molecular and host range data, places $H$. zachvatkini among the most comprehensively defined members of Trombiculidae.
\end{abstract}

Keywords Chigger mites $\cdot$ Morphology $\cdot$ COI $\cdot$ Hosts $\cdot$ Rodents $\cdot$ Phenotypic plasticity

\section{Introduction}

Trombiculidae sensu Goff (1999) comprise ca. 3000 species, with the vast majority (about $90 \%$ ) known exclusively from larvae. Morphology-based methods of species identification, fragmentary knowledge of phenotypic plasticity, scarcity of distributional data, and descriptions based on larvae, make it difficult to evaluate the actual number of species. The difficulties in species delimitation stem also from incomplete knowledge of host spectra and possible host-driven intra-population differences.

Joanna Mąkol

joanna.makol@up.wroc.pl

1 Institute of Biology, Department of Invertebrate Systematics and Ecology, Wrocław University of Environmental and Life Sciences, Kożuchowska 5b, 51-631 Wrocław, Poland 
Despite observed morphological differences among Psoroptes skin mites (Astigmata: Psoroptidae), Pegler et al. (2005) found no molecular evidence of species-level diversity and thus refuted the earlier concept of distinct specific identity of the parasites from different host species. Data on host-associated differences among trombiculids are very scarce. Menezes et al. (2011) failed to find any significant morphological differences between groups of Eutrombicula alfreddugesi (Oudemans), which infested different species of lizards, whereas Kuo et al. (2011) observed differences in the degree of engorgement (inferred from idiosoma length and width) of Leptotrombidium imphalum Vercammen-Grandjean and Langston within and among its three host species.

Hirsutiella zachvatkini, widely distributed in Europe and Asia, is regarded as one of the most common chigger species. Its presumably wide host spectrum includes rodents, insectivores, lagomorphs and birds (Kudryashova 1998). Active postlarval forms of $H$. zachvatkini have been re-described by Daniel (1961). Data on metric and meristic characters of larvae have been provided by Stekolnikov (2001a), who has also dealt with chaetotactic anomalies and intraspecific variation of Hirsutiella spp. (Stekolnikov 2001b, 2003), and also by Imaz et al. (2005), however the host-induced variability was not explicitly examined.

Here we provide the results of morphometric and molecular analyses of larvae of $H$. zachvatkini, collected from striped field mouse, Apodemus agrarius (Pallas) (Muridae), yellow-necked mouse, Apodemus flavicollis (Melchior) and bank vole, Myodes glareolus (Schreber) (Cricetidae). Our study aims at answering the question of potential differences between mites infesting different host species.

\section{Materials and methods}

Ectoparasitic larvae (total: 133 specimens) were collected from A. agrarius (46 larvae/11 host specimens), A. flavicollis (45/10) and M. glareolus (42/9). The hosts were captured in Sherman traps (permissions 66/2012, 27/2013 and 41/2013 issued by the Second Local Commission for Animal Experiments) in a deciduous forest stand in the Syców Municipal Park $\left(51^{\circ} 17^{\prime} 22.672^{\prime \prime} \mathrm{N}, 17^{\circ} 42^{\prime} 39.766^{\prime \prime} \mathrm{E}\right)$, Poland, between September 2012 and April 2014. The larvae were preserved in $96 \%$ ethanol.

A molecular analysis, aiming at evaluating the differences between the examined specimens, was carried out on three larvae and three deutonymphs (reared from the most engorged larvae). Each pair (larva + deutonymph which developed from engorged larva) originated from a different host species. Total genomic DNA was extracted using DNeasy Blood and Tissue Kit (Qiagen). The mites were transferred from $96 \%$ ethanol to ATL lysis buffer with Proteinase $\mathrm{K}$ and incubated overnight at $56{ }^{\circ} \mathrm{C}$. After digestion, the lysis buffer containing nucleic acids was transferred to a new Eppendorf tube and stored for DNA isolation according to the manufacturer's protocol. Amplification of the DNA barcode region (cytochrome c oxidase 1 subunit) was performed using degenerate primers: bcdF04 (5'-CATTTTCHACTAAYCATAARGATATTGG-3') and bcdR04 (5'-TATAAACYTCD GGATGNCCAAAAAA-3 ${ }^{\prime}$ ) (Dabert et al. 2010) with the following thermocycling conditions: $95^{\circ} \mathrm{C} / 3$ min-initial denaturation; $95^{\circ} \mathrm{C} / 30 \mathrm{~s}, 48{ }^{\circ} \mathrm{C} / 30 \mathrm{~s}, 72{ }^{\circ} \mathrm{C} / 45 \mathrm{~s}-40$ cycles; $72{ }^{\circ} \mathrm{C} / 7 \mathrm{~min}$-final extension. The PCR reaction $(25 \mu \mathrm{l})$ was performed using the following PCR mix: $4 \mu \mathrm{l}$ of genomic DNA, $10 \mathrm{mM}$ Tris- $\mathrm{HCl}, 50 \mathrm{mM} \mathrm{KCl}, 1.5 \mathrm{mM}$ $\mathrm{MgCl}_{2}, 200 \mu \mathrm{M}$ of each dNTP, 150 pmol of each primer and 2 units of Taq polymerase (EurX). The amplification product was purified using QIAquick PCR purification kit 


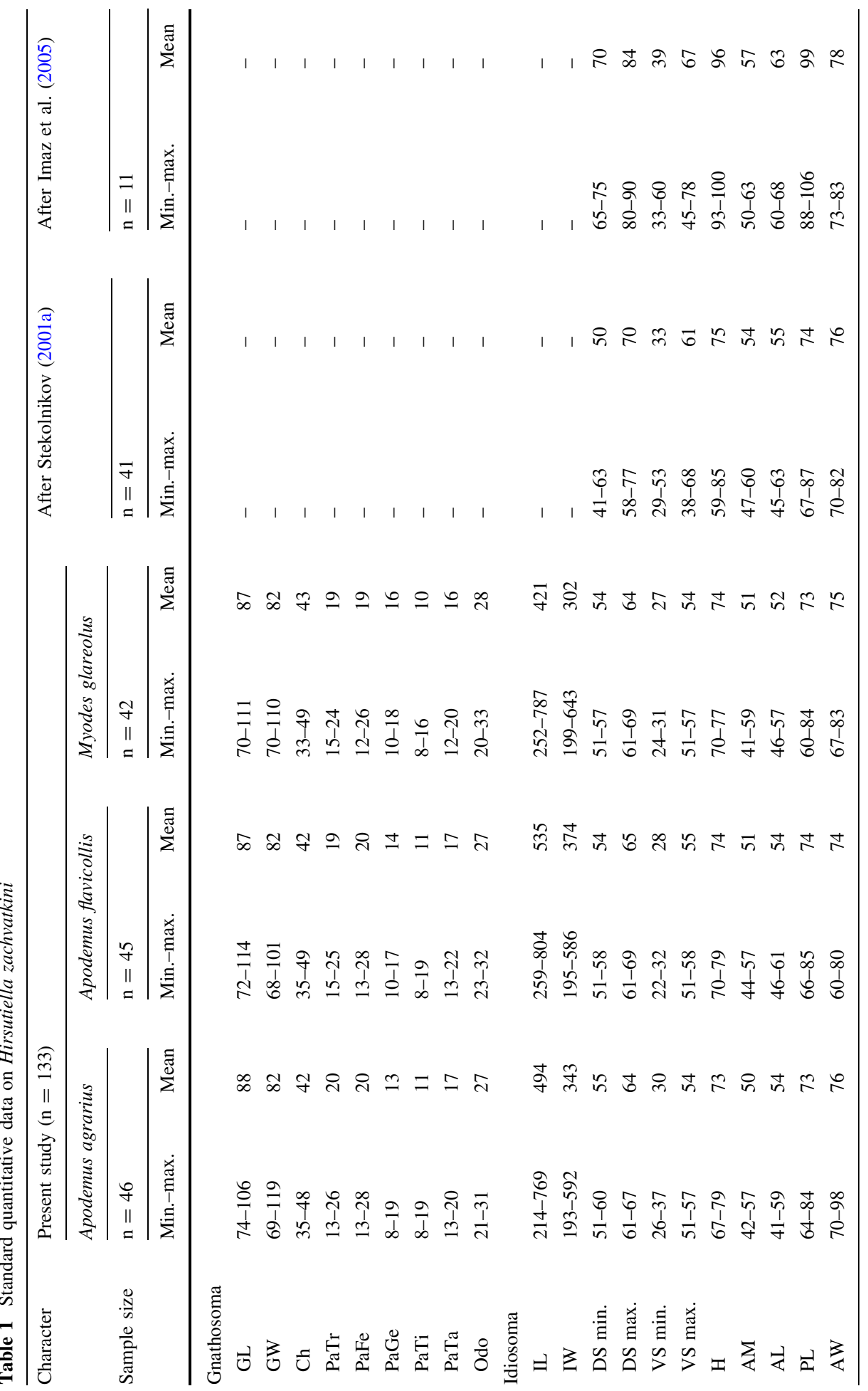




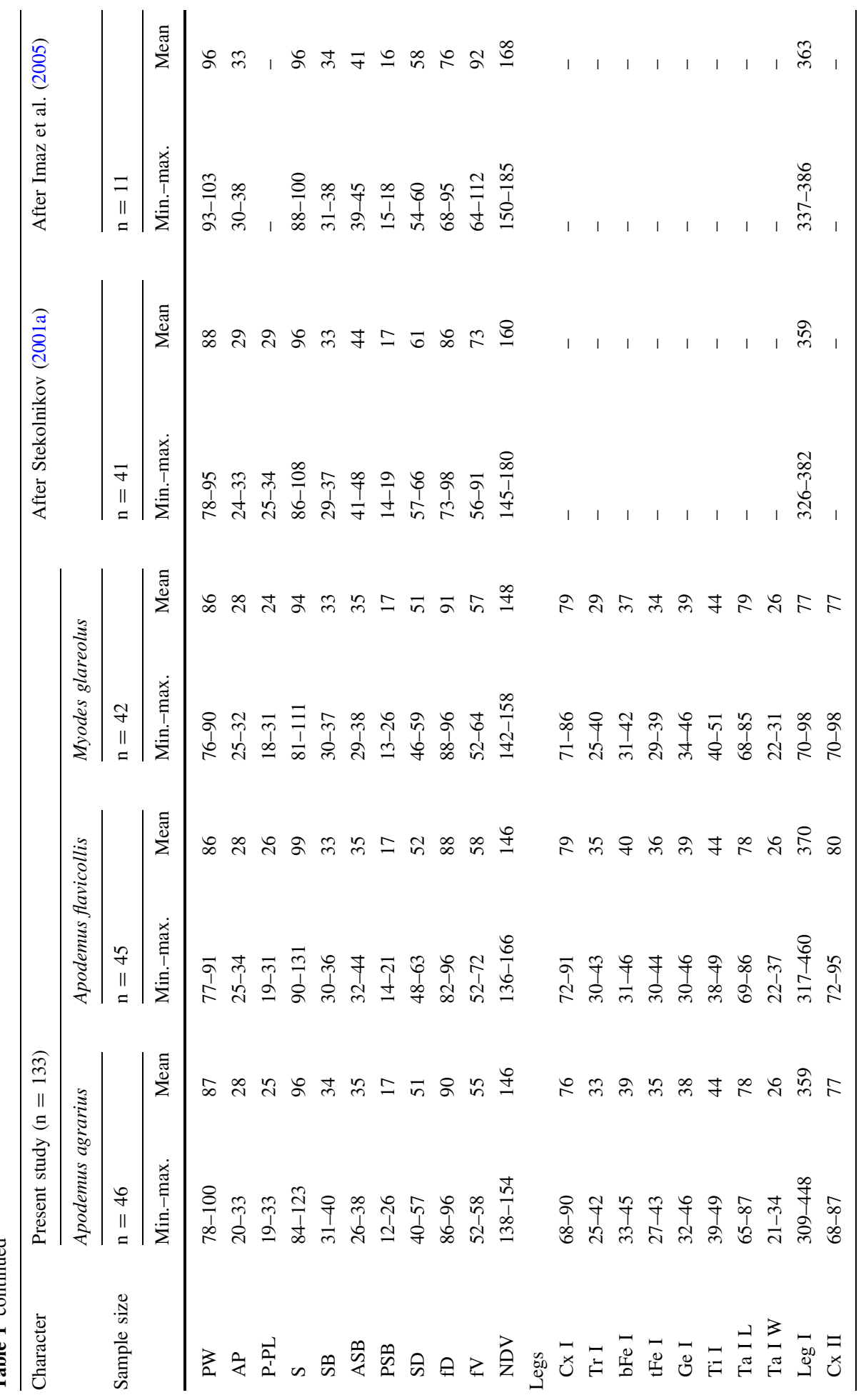




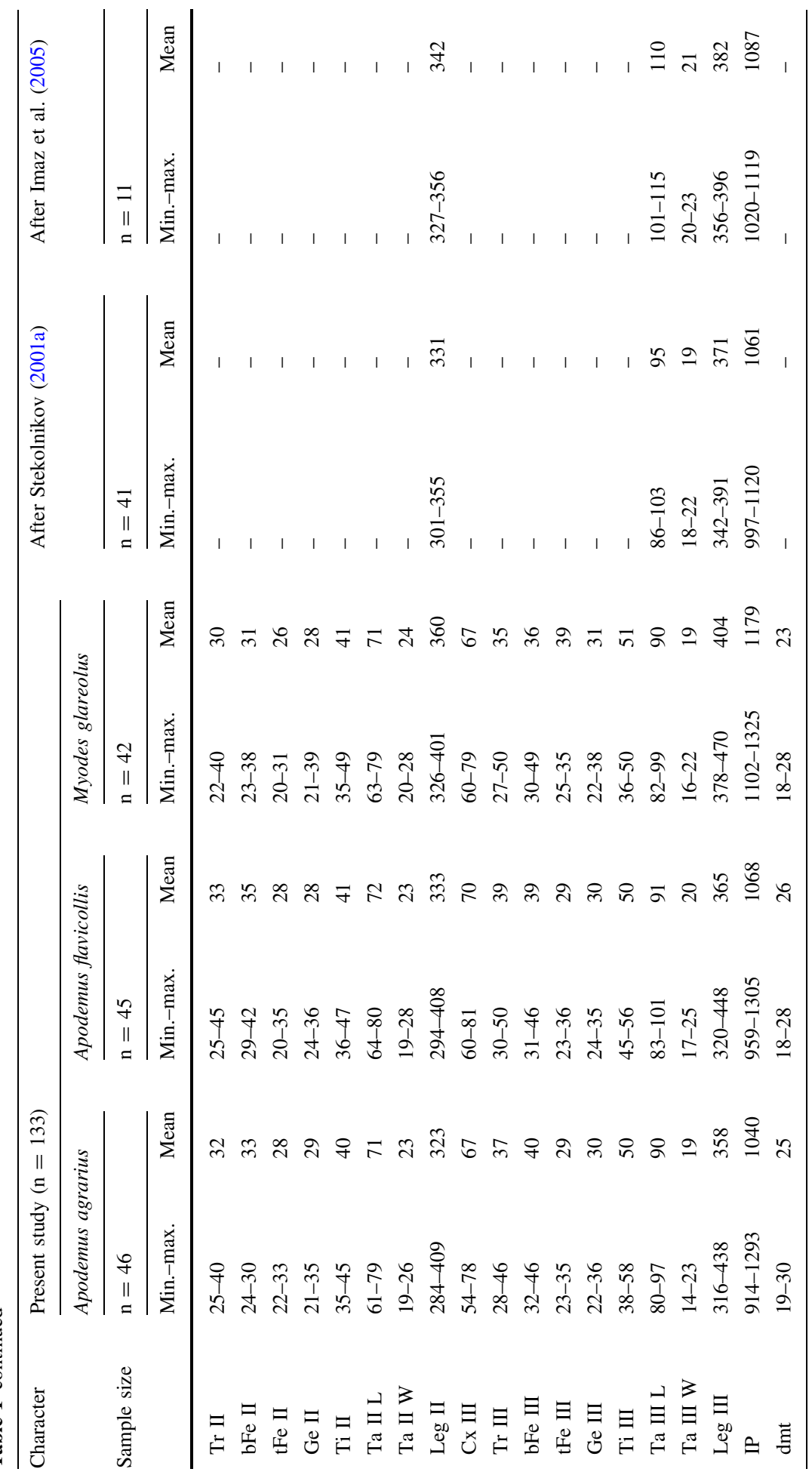




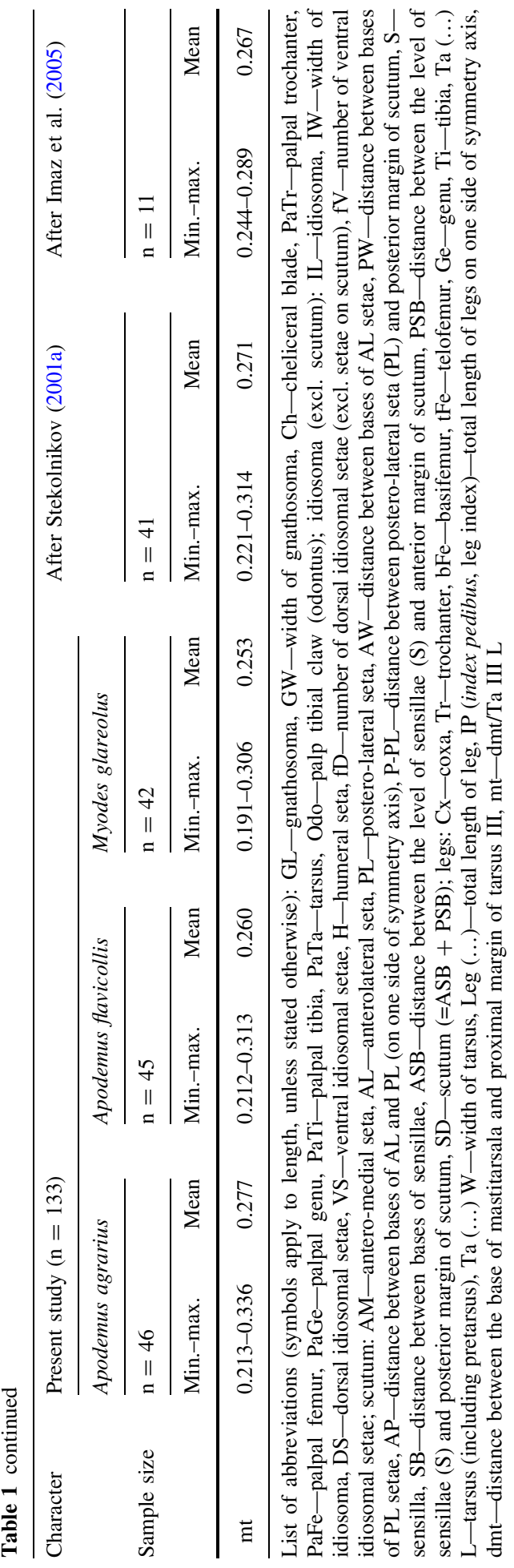


(Qiagen) and sequenced on both strands (Genomed, Poland). The sequences of H. zachvatkini isolated from analyzed host species were identical, thus only one, obtained from deutonymph that developed from larva parasitising the bank vole, was deposited in GenBank (acc. no. KR071845).

Specimens that served for morphological studies (incl. exoskeletons that remained after DNA extraction) were mounted on microscopic slides in Hoyer's medium. Measurements and photos were taken under a Nikon Eclipse E600 compound microscope equipped with DIC and DS-Fi1 camera, using the NIS-Elements BR software. Morphological terminology follows Goff et al. (1982). All the measurements are given in micrometres $(\mu \mathrm{m})$.

Table 2 Summary of discriminant function analysis across the complete list of variables

\begin{tabular}{|c|c|c|c|c|c|}
\hline Variable & Wilks' lambda & Partial lambda & $p$ value & Root 1 & Root 2 \\
\hline $\mathrm{Ch}$ & 0.237 & 0.962 & 0.12 & -0.269 & -0.039 \\
\hline SB & 0.235 & 0.973 & 0.23 & 0.113 & 0.359 \\
\hline AW & 0.240 & 0.952 & 0.070 & -0.151 & 0.490 \\
\hline PW & 0.231 & 0.986 & 0.47 & 0.091 & -0.263 \\
\hline AP & 0.228 & 0.999 & 0.96 & -0.012 & -0.055 \\
\hline ASB & 0.233 & 0.980 & 0.33 & -0.055 & -0.286 \\
\hline AM & 0.229 & 0.996 & 0.79 & 0.043 & -0.118 \\
\hline PSB & 0.242 & 0.944 & 0.045 & 0.103 & -0.451 \\
\hline $\mathrm{AL}$ & 0.239 & 0.956 & 0.090 & 0.298 & -0.118 \\
\hline PL & 0.231 & 0.989 & 0.56 & -0.045 & -0.193 \\
\hline $\mathrm{S}$ & 0.248 & 0.920 & 0.011 & 0.341 & -0.271 \\
\hline PaTr & 0.229 & 0.997 & 0.85 & -0.042 & 0.093 \\
\hline $\mathrm{PaFe}$ & 0.237 & 0.963 & 0.13 & 0.250 & 0.149 \\
\hline $\mathrm{PaGe}$ & 0.232 & 0.982 & 0.39 & 0.014 & -0.245 \\
\hline PaTi & 0.258 & 0.884 & 0.001 & 0.478 & 0.268 \\
\hline PaTa & 0.266 & 0.859 & $<0.001$ & 0.479 & -0.290 \\
\hline Odo & 0.237 & 0.965 & 0.14 & -0.261 & 0.107 \\
\hline Leg I & 0.241 & 0.949 & 0.059 & -0.641 & -0.051 \\
\hline Leg II & 0.234 & 0.976 & 0.26 & 0.252 & -0.610 \\
\hline Leg III & 0.243 & 0.941 & 0.038 & -0.731 & 0.475 \\
\hline $\mathrm{dmt}$ & 0.243 & 0.938 & 0.032 & 0.272 & 0.327 \\
\hline $\mathrm{fV}$ & 0.233 & 0.978 & 0.31 & 0.063 & -0.261 \\
\hline fD & 0.238 & 0.958 & 0.096 & 0.033 & 0.439 \\
\hline \multicolumn{4}{|l|}{ Eigenvalue } & 1.824 & 0.551 \\
\hline \multicolumn{4}{|c|}{ Cumulative proportion } & 0.768 & 1.000 \\
\hline Roots removed & Eigenvalue & Wilks' lambda & Chi square & df & $p$ value \\
\hline 0 & 1.825 & 0.228 & 175.779 & 46 & $<0.001$ \\
\hline 1 & 0.551 & 0.645 & 52.215 & 22 & $<0.001$ \\
\hline
\end{tabular}

Chi square tests with successive roots removed are provided in the lower part of the table. Root 1 and Root 2 columns refer to standardized coefficients of canonical variables 
The larvae were preliminarily assigned to $H$. zachvatkini based on morphological criteria (Kudryashova 1998; Stekolnikov 2001a).

Our morphological analysis identified 61 characters of the gnathosoma, idiosoma and legs. For the list of characters and explanation of symbols see Table 1. Statistical analysis was carried out using Statistica 10 software (StatSoft 2011). Prior to the analysis the data were $\log$-transformed $\left(\log _{10}\right)$. Mean and minimum/maximum values for all variables were calculated. Out of 61 morphological characters, 23 (Ch, SB, AW, PW, AP, ASB, AM, PSB, AL, PL, S, PaTr, PaFe, PaGe, PaTi, PaTa, Odo, Leg I, Leg II, Leg III, dmt, fV, fD) were selected for discriminant function analysis (DFA). Since some of the characters listed in Table 1 were not independent, we preselected the variables and DFA was restricted to those, which were measured directly.

\section{Results}

Sequencing of the COI gene yielded six identical barcode sequences of $680 \mathrm{bp}$. We did not observe any nucleotide substitutions in this region, and no intraspecific variation at molecular level could be confirmed.

The ranges of larval characters used in the present study and those examined by Stekolnikov (2001a) and Imaz et al. (2005) overlapped (Table 1), except for DS min., DS max., H and PL provided by Imaz et al. (2005), hence, in the lack of other differentiating characters, the affiliation of our material with T. zachvatkini could be confirmed.

The model generated by DFA is provided in Table 2. The Roots 1 and 2 account for 76.8 and $100 \%$ of the total variation within H. zachvatkini collected from three host species. The variables that play the major role in this differentiation are, in descending order, Leg III, PaTa, PaTi, S, dmt and PSB. The means of canonical values (Table 3) indicate that Root 1 discriminates the specimens of $H$. zachvatkini obtained from $M$. glareolus. When the canonical scores from the discriminant analysis are plotted and viewed (Fig. 1), it can be seen that representatives of $H$. zachvatkini collected from $M$. glareolus [with the total percentage of correctly classified specimens accounting for $95.2 \%$ (Table 3)] are clearly separated from the mites collected from Apodemus mice.

\section{Discussion}

Identical DNA sequences obtained from the chigger specimens parasitising different host species suggest their conspecificity. This is compatible with Shatrov and Kudryashova's (2008) view that host selection in trombiculid mites is imposed by the habitat of the larvae,

Table 3 Classification efficiency of Hirsutiella zachvatkini from each host species

\begin{tabular}{lllllll}
\hline & $\begin{array}{l}\text { \% of correct } \\
\text { classification }\end{array}$ & $\begin{array}{l}\text { A. agrarius } \\
(p=0.35)\end{array}$ & $\begin{array}{l}\text { A. flavicollis } \\
(p=0.34)\end{array}$ & $\begin{array}{l}\text { M. glareolus } \\
(p=0.32)\end{array}$ & Root 1 & Root 2 \\
\hline A. agrarius & 78.3 & 36 & 6 & 4 & 1.019 & 0.839 \\
A. flavicollis & 71.1 & 8 & 32 & 5 & 0.788 & -0.930 \\
M. glareolus & 95.2 & 0 & 2 & 40 & -1.961 & 0.077 \\
Overall & 81.2 & 44 & 40 & 49 & & \\
\hline
\end{tabular}

Root 1 and Root 2 columns refer to the means of canonical values 


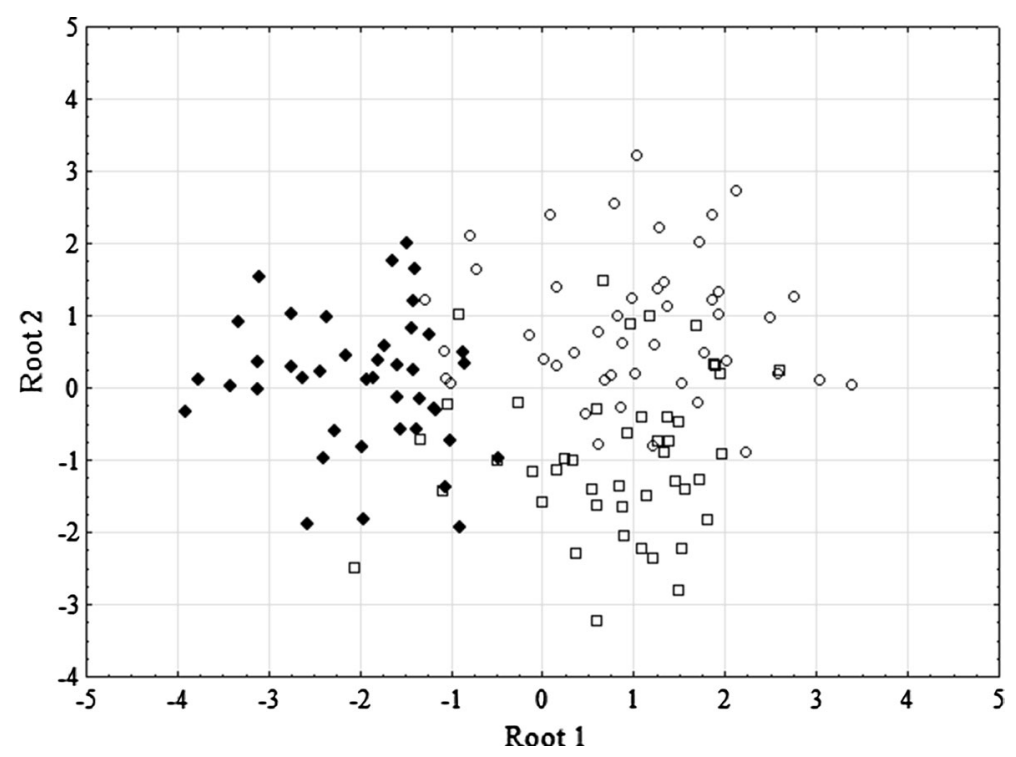

Fig. 1 Results of canonical analysis of Hirsutiella zachvatkini obtained from three host species. Plot generated based on 23 variables measured in 133 specimens. Symbols denoting host species: squaresApodemus flavicollis, circles-Apodemus agrarius, black diamonds-Myodes glareolus

which infest all available vertebrates. According to Stekolnikov and Klimov (2010) size variation may reflect differences in environmental conditions, and is not necessarily genetically-based, as opposed to qualitative traits. As stated by Traub and Wisseman (1974) trombiculid larvae during their search for host are exposed to desiccation, therefore the risk of failure in finding the suitable host makes them less host-selective, even in view of lower energetic returns (Kuo et al. 2011).

The identical COI sequences may also reflect a relatively short co-evolution of parasites and their hosts. The assumption is especially relevant in the case of parasitengone mites, which may have switched their host groups several times; for example, between insects or from insects to arachnids and, in the case of Trombiculidae, to vertebrates (Audy 1960). The wide host range and the distribution of hosts on the phylogenetic tree of parasitengone mites do not allow an exact determination of the primary host or host range in the stem lineage of the cohort (Wohltmann 2000). No doubt, vertebrate hosts offered new evolutionary possibilities and reduced the selection pressure through their morphological constitution, body mass and continuous abundance throughout the year (Wohltmann 2000). The strategy, which may reflect similar evolutionary trends, has been already recognised in other, non-chigger, mites. Baulechner's et al. (2013) morphological and molecular (COI) analysis of host specificity in three species of Spinturnix (Mesostigmata: Spinturnicidae) parasites of four sympatric bat species (Myotis spp.) revealed the occurrence of three major, morphologically different clades. Yet, there was no evidence for co-speciation, but host switch and sorting event were confirmed. Furthermore, the hosts were several million years older than their parasites.

The morphological differences among the chiggers collected from the bank vole and mice should be regarded as intraspecific variation, which is induced by the host and thus reflects the adaptation to local microenvironment. Pegler et al. (2005) studied putative 
species of Psoroptes (Psoroptidae), associated with different host taxa. Their morphological and molecular (ITS-2 gene sequence) analyses showed that the observed variation was insufficient to consider the mites as representing distinct species. Nevertheless, the conclusions of Pegler et al. (2005) and those resulting from our study are not supported by the same strategies involved in host-parasite associations. In the case of psoroptids, the whole life cycle occurs on the host, whereas in Trombiculidae the contact with the host is limited to the larva only. The different selection pressures on the larval instar may be important for further conclusions. Contrary to most other parasitengones, the duration of contact with the host in trombiculids may go beyond the actual phase of parasitism. Traub et al. (1975), observed larvae of Leptotrombidium spp. associated with their hosts (op. cit. "chiggers would wander for hours, or a day or longer") before the onset of feeding. The latter, besides the knowledge of local adaptation of larvae to occupy particular places within the host body, may contribute to finding the background for the most pronounced morphological differences in the length of leg segments among the chiggers collected from the bank vole and mice. It cannot be excluded that during the prolonged contact with host, the neosomy, i.e. additional production of cuticle without intermittent moult, the phenomenon described by Audy et al. (1972) for Vatacarus (Trombiculidae) and reported also by Wohltmann (1999) in relation to non-trombiculid parasitengone mites, may occur. As opposed to legs, we observed a relatively small and statistically insignificant variation of the morphological characters of the scutum. The lack of differences in scutal traits is compatible with the results obtained by Menezes et al. (2011) for Eutrombicula alfreddugesi collected from various lizard species. The authors compared six metric characters of the scutum in E. alfreddugesi collected from four species of Tropidurus spp. (Reptilia: Squamata).

Despite the fact that further studies should focus on retracing the ecological background and consequences of host-parasite association, the present knowledge of variation of metric characters in larvae of $H$. zachvatkini (Table 1), supported by qualitative and meristic characters, molecular data and host range data, allows to place the species in question among the most comprehensively defined members of Trombiculidae.

Acknowledgments The study was financed by the National Science Centre, Poland, Project No. DEC2012/07/N/NZ8/02354.

Open Access This article is distributed under the terms of the Creative Commons Attribution 4.0 International License (http://creativecommons.org/licenses/by/4.0/), which permits unrestricted use, distribution, and reproduction in any medium, provided you give appropriate credit to the original author(s) and the source, provide a link to the Creative Commons license, and indicate if changes were made.

\section{References}

Audy JR (1960) Evolutionary aspects of trombiculid mite parasitism. In: Purchon RD (ed.) Proceedings of the century and bicentury Congress of Biology. University of Malaya press, Singapore, pp 102-108

Audy JR, Radovsky FJ, Vercammen·Grandjean PH (1972) Neosomy: radical intrastadial metamorphosis associated with arthropod symbiosis. J Med Entomol 9:487-494

Baulechner D, Becker NI, Encarnaçāo J (2013) Host specificity in spinturnicid mites: do parasites share a long evolutionary history with their host? J Zool Syst Evol Res 51:203-212

Dabert M, Witaliński W, Kaźmierski A, Olszanowski Z, Dabert J (2010) Molecular phylogeny of acariform mites (Acari, Arachnida): strong conflict between phylogenetic signal and long-branch attraction artifacts. Mol Phylogenet Evol 56:222-241 
Daniel M (1961) Contribution a la connaissance des formes adultes des Trombiculidae d'Europe. I. Description des nymphes et des adultes du Trombicula (N.) zachvatkini Schluger 1948 et Trombicula (N.) talmiensis Schluger 1955. Acarologia 3:24-47

Goff ML (1999) The current state of chigger systematics: a view from a swamp $20 \mathrm{~km}$ SSE of Eden. In: Needham GR, Mitchell R, Horn DJ, Welbourn WC (eds) Acarology IX, vol 2., Symposia Ohio Biological Survey, Columbus, pp 145-149

Goff ML, Loomis RB, Welbourn WC, Wrenn WJ (1982) A glossary of chigger terminology (Acari: Trombiculidae). J Med Entomol 19:221-238

Imaz A, Galicia D, Moraza ML, Stekolnikov AA (2005) Contribution to the knowledge of chigger mites (Acari: Trombiculidae) parasitizing Apodemus sylvaticus (L.) (Rodentia, Muridae) on the Iberian Peninsula. Acarologia 46:53-64 (2006)

Kudryashova NI (1998) Chigger mites (Acariformes, Trombiculidae) of East Palearctics. KMK Scientific Press, Moscow, p 342 (In Russian)

Kuo C-C, Wang H-C, Huang C-L (2011) Variation within and among host species in engorgement of larval trombiculid mites. Parasitology 138:344-353

Menezes VA, Fontes AF, Gettinger D, Van Sluys M, Rocha CFD (2011) A morphometric study of Eutrombicula alfreddugesi (Acari: Trombiculidae) infesting four sympatric species of Tropidurus (Squamata: Tropiduridae) in northeastern Brazil. Phyllomedusa 10:79-84

Pegler KR, Evans L, Stevens JR, Wall R (2005) Morphological and molecular comparison of host derived populations of parasitic Psoroptes mites. Med Vet Entomol 19:392-403

Shatrov AB, Kudryashova NI (2008) Taxonomic ranking of major trombiculid subtaxa with remarks on the evolution of host-parasite relationships (Acariformes: Parasitengona: Trombiculidae). Ann Zool 58:279-287

StatSoft (2011) STATISTICA (data analysis software system), version 10. www.statsoft.com

Stekolnikov AA (2001a) Systematics of chigger mites of the genus Hirsutiella Schluger et Vysotzkaya, 1970 (Acari: Trombiculidae). Entomol Rev 80:219-242 (In Russian, English summary)

Stekolnikov AA (2001b) Intraspecific variance of chaetotactic characters in the chigger mite genus Hirsutiella (Acari: Trombiculidae). Parazitologiya 35:19-26 (In Russian, English summary)

Stekolnikov AA (2003) Intraspecific variability and sympatria in closely related chigger mites species of the genus Hirsutiella (Acari: Trombiculidae). Parazitologiya 37:281-297 (In Russian, English summary)

Stekolnikov AA, Klimov P (2010) A revision of chiggers of the minuta species-group (Acari: Trombiculidae: Neotrombicula Hirst, 1925) using multivariate morphometrics. Syst Parasitol 77:55-69

Traub R, Wisseman CL Jr (1974) The ecology of chigger-borne rickettsiosis (scrub typhus). J Med Entomol 11:237-303

Traub R, Wisseman CL, Jones MR, O'Keefe JJ (1975) The acquisition of Rickettsia tsutsugamushi by chiggers (trombiculid mites) during the feeding process. Ann N Y Acad Sci 266:91-114

Wohltmann A (1999) Life history evolution in Parasitengonae (Acari: Prostigmata): constraints on number and size of offspring. In: Bruin J, van der Geest LPS, Sabelis M (eds) Evolution and ecology of Acari. Kluwer, Dordrecht, pp 137-148

Wohltmann A (2000) The evolution of life histories in Parasitengona (Acari: Prostigmata). Acarologia 41:145-204 (2001) 